\title{
Research on Status and Development of Jet Pump
}

\author{
Zhengyang $\mathrm{An}^{1, \mathrm{a}^{*}}$ \\ ${ }^{1}$ Kunming Fire Services Training School, \\ Kunming China \\ a anzhengyang2005@sina.com
}

\author{
Weiguo Liang ${ }^{2}$ \\ ${ }^{1}$ Kunming Fire Services Training School, \\ Kunming China \\ 1079727091@qq.com
}

\begin{abstract}
Jet pump is a mechanism of vaccum pump working with medium of liquid or gas for the transportation of fluids such as solid, liquid and gas in the fields of water conservancy, power, petroleum, metallurgy, transportation, chemical industry, environmental protection and fire protection. In this paper, the basic structure and working principle of jet pump were discussed firstly, and then the research status of jet pump was presented from points of design theory, fluid inner flowing and practical application of jet pump. Based on the deep analysis of the development trend of jet pump in future, this article has certain reference value for future research.
\end{abstract}

Keywords-jet pump; features; development status; trend; theoretical basis

\section{INTRODUCTION}

Jet jump is a machine with working medium (liquid or gas) to transport fluid. The characteristic of jet pump is that the jet pump with simple structure and without any moving part has reliable performance and good sealing. It has been applied widely under special working environments of high temperature, high pressure, in vacuum and underwater. The energy of low-speed absorbed liquid can be improved by high-speed flowing liquid in the jet pump. Working with displacement pump or vane pump, the suction lift of the whole system and suction performance can be improved greatly.

The basic structure of jet pump is shown in Fig .1.. Jet pump is composed of nozzle, throat and diffuser. The working principle is that when working liquid blowing out through the nozzle at a high speed, the static energy of the working fluid turns into the kinetic energy of the absorbed liquid. The pipe in a vaccum absorbs the low-pressure liquid. Two kinds of fluids mix up in the throat and exchange their energy, and the speed of the working fluid will decrease, while the absorbed liquid speed will rise, both reaching almost equivalent at the output of the throat. When the fluid flows through the diffuser, the speed of the mixed fluid decreases gradually with the pipe diameter, and the kinetic energy transforms into pressure energy, resulting the pressure increase of the mixed fluid.

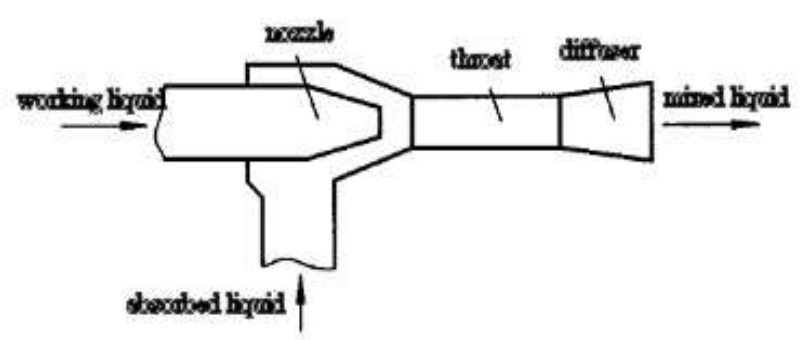

Figure 1. The basic structure of a jet pump

II. Research status of jet pump

A. Research status of design theory

At the beginning of 1860s, Zeuner built the basic design theory of jet pump. After this, he developed and perfected the theory furtherlly with Rakine[1]. But the disadvantage of their theory is that it failed to solve the theoretical calculation of jet pump. Until 1930s, with the development of fluid mechanics and aerodynamics, the design theory of jet pump developed to be mature gradually. In 1933, basic performance equations about jet pump were set up after series systematic work of Gosline[2]. In 1952, the performance calculation method of jet pump was afforded by Maconaghy[3]. After detail experiments on jet pump of water and water vapor, 
Bonnigton put forward the reasonable sizes for each part of jet pump[4].In 1965, Hansen presented the design method of liquid jet pump. Hongyu Lu figured out the design theory and design method of various jet pumps, which set up a solid foundation for further research[6].

Recently, many scholars focused on some particular jet pumps and put forward many new design theories. After researching about waste-gas jet pump, Chuanjnu Li [7] proposed that the design of jet pump and the main working parameters of jet pump can be calculated using a method based on the isentropic flow of single gas phase. Using plane potential flow theory, Fang Zhang finished the theoretical design and research work of those working parameters of concrete jet pump and set up a new set of method for the design of jet pump.

The core problem of jet pump is to increase the efficiency of jet pump effectively. However, the efficiency of jet pump is relatively low due to the loss of energy in the process of mixing. Therefore, the design of jet pump and the optimization of working parameters have been the focus of the research for years.

According to the research, there are two approaches to improve the efficiency of jet pump. One is to optimize the parameters through the structure improvement of the jet pump. Winoto[9] obtained by theoretical design and experiment that jet pump had the highest efficiency when the ratio of the area of nozzle to that of the throat was 0.3 . After introducing three parameters: width ratio of pipe to nozzle, pressure ratio at nozzle and Reynolds Number, After analyzing the 2D-flow field with N-S Equation, Ning and Satofuka believed that there was the optimum width ratio of pipe to nozzle for the highes efficiency of jet pump.

Changbin Wang[11] researched the optimization of the jet pump parameters using energy conservation principle and multivariate function extreme value theory. The result showed that under conditions of isodensity system, a certain friction loss coefficient and the optimal area ratio 0.28 , the efficiency of jet pump was the highest. Friction loss coefficient was the main influence on the efficiency. Optimal area ratio was only related to the friction loss coefficient and had nothing to do with the density ratio of different fluids.

Aiguo Liang[12] calculated the jet pump performance with different area ratios, With efficiency as the evaluation scale, he concluded that when the area ratio ranging from 3.0 to 3.8 , the optimum throat length of the jet pump can be calculated by equation as follows:

$$
\mathrm{L}=(0.222+5.6154) \times \mathrm{d} 3
$$

where $\mathrm{d} 3$ as throat diameter.

Another approach to improve the efficiency is to use unsteady jet, such as pulsed jet and concussion jet on the jet pump with the same structure[13]. Hongyi Lu investigated the pulse centrifugal jet vacuum pump and researched its running mechanism, and put forward the basic equation. Since 1994, Xinping Long have carried out series of basic research on pulse liquid jet pump. The result showed that the efficiency of pulse liquid jet pump was higher than that of steady flow jet pump. In 1995, Chuanchang Gao mastered preliminarily the stably working conditions of gas-liquid Piston pulse jet pump with different geometry parameters and working parameters of nozzle aperture and area ratio, length between throat and nozzle pulse frequency.

In summary, the study of all aspects of unsteady jet especially pulse jet is still not deep, basically staying on prototype testing stage. Although many experiments proved that the energy transfer efficiency of pulse jet pump was higher than that of steady jet pump, both the design theory and the flow mechanism of pulse jet pump need further study.

\section{B. Internal flow study}

Because of the fast development of testing method and appearance of CFD software, many researchers are able to study the flow field to reveal the flow law of liquid in the jet pump.

On the aspect of liquid testing in the jet pump, the preferred methods for testing liquid are speed-pressure probe, hot wire anemometer, laser velocimeter. However, those methods have a common defect that they are only used for spot measurement instead of simultaneous 
measurement in the whole flow field. To solve this, Aiguo Liang[14] measured the flowing situation of the whole flow field in the jet pump at any time with advanced PIV technology and analyzed the flow field in the throat. The results showed that flow structure was divided into nuclear area, jet boundary layer zone, recirculation zone and pipe flow area. Under condition of a certain jet pump structure (the area ratio of nozzle to throat was certain), the flow structure was only related to flow ratio. When flow ratio was large, jet decreased slowly and the nuclear area became longer. When flow ratio was low, jet decreased quickly, the nuclear area became shorter reflux appeared, and efficiency decreased. Using the feature that jet pump have certain flow ratio on the condition of cavitation, Lei Gu[15] studied the effect of shape and working parameters on jet pump cavitation flow-rate ratio and system efficiency. Through contrast test of the work performance between first-grade jet pump and double-grade jet pump, they determined the appropriate ratio scheme.

Numerical Simulation is to analyze the system of liquid flow heat conduction and other related physical phenomena through numerical calculation and images with computer to obtain basic physical quantities of all location, such as speed, pressure and temperature, and their changes with time in an extremely complicated flow field,. Correct simulation can guide design of fluid mechanical, avoid tedious model experiment, reduce the design cost and provide good theory basis for experimental research. The method applied the most often is using FLUENT software to carry on three dimension value simulation in the flow field of jet pump with N-S equation combining $\mathrm{k}-\varepsilon$ realizable turbulence model. Hongjun Chang[16] obtained the distributions of the speed and the pressure of jet pump in the 3D flow field with this method. Changbin Wang[17] concluded through numerical simulation that axial speed profile of flow field hadgood self-similarity in segment of diffuser instead of in throat, whose kickpoint was related to the length of throat. The turbulent Kinetic energy of the flow field had peaks at the nozzle exit and diffuser entrance, respectively, and the former was much higher than the latter. Therfore, as for a jet pump flow field, turbulence mainly occurs in the throat entrance and Imbalance of turbulent Kinetic energy causes extra energy loss. Tongzhuo Li [18] carried out numerical simulation of inner flow field of jet pump. From the view of velocity vector, centre line velocity diagram and speed distribution of several sections, the result of simulation was accord with engineering practice. This method becomes a new road for future research on numerical simulation.

\section{Basic Application Research}

In twentieth Century sixty time, in order to solve the problem of groundwaterextraction of Hexi Corridor in Gansu Province, chinese experts combined jet pump with centrifugal pump to research the jet pump. Because of the adding of the jet pump, the whole suction is equal to the centrifugal pump and jet pump suction. So the equipment can Pumping from deep wells more than 10m, which replace the submersible pump.

Jet pump is used widely in compression and improvement of cavitation performance. Chunwang Liu[19] applied venturi tube jet pump device to recycle low-pressure gas. The device compressed vaporizing gas from the oil-water tank at medium pressure, and transported to gas transmission system for different use. Besides, it can be used to recycle the gases discharged from glycol dehydrator, heating processor and low pressure separator. In May 15, 2002, the equipment is used to explore the oil and gas use in the E1Ebanito at Texasoil in USA, which can earn revenue more than $\$ 33600$ from the gas recovery volume every day.

To solve the noise and vibration produced on the inlet and outlet of the pipeline in the large carbonate pump units, Fanyu Kong[20] added jet device at the entrance of pump pipeline, introducing a part of high pressure liquid in the pump exit into the jet device to mix up with the liquid from the tank. The available npsh of the pump was improved through supplying the pump entrance with 
increased pressure liquid and the above problems were solved effectively.

Jianrui Liu[21] add a jetted self-priming set with a ventri to a gasline engine pump. When fluid at high pressure jetted at high speed from the chamber to the pump entrance through the nozzle, a strong suction vacuum was produced to start the common pumps by self suction. In the process of the pump running, while the self-circulation process can be achieved, the valve of the self-circulation jetted set can be closed. The performance test of the pump demonstrated that the pump could complete the self-circulation process within a shorter time, while $\mathrm{H} \sim \mathrm{Q}$ curve was stable and flat and the efficiency was $3 \%$ higher than that of the conventional centrifugal pumps. So high speed liquid flow of the jet pump nozzle can form a low voltage and effectively shorten the time of self - priming when it is applied to the self suction centrifugal pump.

The Development Trend of Jet Pump

After analyzing the design theory, inner liquid flow law and application of jet pump, it is predictable for the future development trend of jet pump:

It is necessary to develop the combining use of jet pump with other kinds of pumps or device to satisfy requirement of different work environment.

Advanced test device or technology should be applied for the deep research on jet pump inner flow liquid flow law to supply reliable experiment basis of theory analysis and actual application.

The relationship of jet pump structural parameters should be investigated to improve the efficiency of jet pump effectively.

By the reasonable design means, a variety of new mechanism and structure of jet pump should be developed Widely to improve furtherly its overall performance.

\section{REFERENCE}

[1] J. M.Rankine. On the mathematical theory of combinedstreams[M]. Landon : Tuwert, 1870: 24-26.

[2] J. E. Gosline, Obfien M. P., Obfien The water jet pump[M]. California; Hewron, 1934: 57-60.

[3] J. W. Maeonaghy. Centrifugal jet pump combinations[M]. Berlin: Oncral, 1952: 12-14.

[4] S. T. Bonnington. Water driven air ejectors[M]. Munich: Uezreos, 1960: 43-44.

[5] A. G. Hansen, R. Kinnavy . The design of water jet pumps[M]. London: Freehous, 1965: 21-23.

[6] Hongyi Lu. The theory and application of jet pump technology[M] Beijing: Waterpower and Electric Power publishing house, 1989: $1-10$.

[7] Chuanjun Li, Weidong Shi, Weidong cao. The principle and design of waste gas jet self-priming device[J]. Pump Technology, 2006(1): 13-4.

[8] Fang Zhang, Jian Yang. Concrete jet pump device theory design[J]. Fluid Machinery, 2000, 28(2): 26-28.

[9] S. H. Winoto, H. Li, D. A. Shah. Efficiency of jet pumps[J]. Journal of Hydraulic Engineering, 2000, 126(2): 150-156.

[10] T. Ning, N. Satofuka, K. G. R. Nippon[J]. B Hen/Transactions of the Japan Society of Mechanical Engineers, Part B, 1997, 63(608) : 1243-1250.

[11] Changbin Wang. The determination method of jet pump best parameter [J]. Fluid Machinery, 2004, 32(9): 21-25.

[12] Aiguo Liang, Jingzhi Liu, Xinping Long. Numerical simulation and throat optimization[J]. Pump Technology, 2003(1): 3-6.

[13] Linghua Wang, Chuanchang Gao. Pulse jet pump research progress $[\mathrm{J}]$. Waterpower \& Electric Power and Machinery, 2006, 28(6): 33-35.

[14] Aiguo Liang, Xinping long, Peijie He. Using PIV to measure jet pump inner flow field [J]. Pump Technology, 2004(1): 10-14.

[15] Lei Gu, Jingsong Zhang, Chunmin Yang. Experiment of cavitation condition liquid jet pump [J]. Fluid Machinery, 2006, 34(2): 7-9.

[16] Hongjun Chang, Yi Zhu. The numerical simulation of jet pump internal 3D flow field[J]. Drainage and Irrigation Machinery, 2005, 23(6): 13-15.

[17] Changbin Wang, Jianzhong Lin, Xing Shi. Numerical simulation and experiment research of jet pump turbulence field $[\mathrm{J}]$. University Chemical Engineering Science Paper, 2006, 20(4): 175-179.

[18] Tongzhuo Li, Bangmin Zheng, HongYi Lu. Using Monte Carlo method to have numerical simulation on inner flow field of jet pump model[J]. Journal of North China Water Conservancy and Hydropower College, 2005, 26(3): 42-44.

[19] Chunwang Liu, Xinquan Liu, Lianjun Ma. Using venturi jet device to collect gas in the tank [J]. Petroleum Machinery, 2005, 33(2): 64-65.

[20] Fanyu Kong, Gang Chen, Jianrui Liu. Using jet device to eliminate cavitation vibration of large pump units[J]. Journal of China Safe Science, 2005, 15(4): 62-65.

[21] Jianrui Dong, Weidong Shi, Zhongming Ye. Research of centrifugal pump jet self-priming device[J]. Agricultural Engineering, 2006, 2(1): 89-92. 